\section{Highlights from}

\section{recent literature}

\section{Analytical}

\subsection{Determination of Size and Concentration of} Gold Nanoparticles from Extinction Spectra

Extinction spectra of colloidal gold can be used for a simple and fast detection of the size and concentration of nanoparticles. It is generally accepted that experimental correlations of the particle size and concentration with the plasmon resonance properties are in agreement with Mie theory simulations. N Khlebtsov, G. Nikolai from the Institute of Biochemistry and Physiology of Plants and Microorganisms, Russian Academy of Sciences, Saratov, Russia, Analytical Chemistry (Washington, DC, United States) 2008, 80(17), 6620. Here, this point in the context of a long-term collection of published experimental data and the T-matrix simulations, which account for deviations of the particle size from ideal monodisperse spheres are discussed. These deviations result in small but quite evident disagreements between the Mie calculations and the experimental calibration curves particle size vs. resonance wavelength. The authors present a long-termaveraged anal. particle-size calibration and also discuss the effects of the particle dielectric functions, shape and size polydispersity on simulated correlations between the extinction spectra and the average particle size, and concentration.

\subsection{Structural, 197Au Mossbauer and Solid State 31P CP/Mas NMR Studies on Bis(Cis-Bis(Dipheny Iphosphino)Ethylene) Gold(I) Complexes [Au(Dppey)2]X For X = Pf6, I}

197Au Mossbauer spectra for the d10 $\mathrm{Au}(I)$ phosphine complexes, [Au(dppey)2]X (X = PF6, I; dppey = cis-bis(dip henylphosphino)ethylene), and the single crystal $x$-ray structure and solid state 31P CPMAS NMR spectrum of [Au(dppey)2]I are reported here. P Healy, B Loughrey, C Bowmaker, J Hanna from School of Biomolecular and Physical Sciences, Griffith University, Nathan, QLD. 4111, Australia, Dalton Transactions 2008, (28), 3723. In [Au(dppey)2]I the AuP4 coordination geometry is distorted from the approx. D2 symmetry observed for the PF6- complex with Au-P bond lengths 2.380(2)-2.426(2) .ANG. and inter-ligand P-Au-P angles 110.63(5)-137.71(8). degree.

Quadrupole splitting parameters derived from the Mossbauer spectra are consistent with the increased distortion of the AuP4 coordination sphere with values of
1.22 and $1.46 \mathrm{~mm} \mathrm{~s}-1$ for the PF6- and I- complexes, respectively In the solid state 31P CP MAS NMR spectrum of [Au(dppey)2]I, signals for each of the four crystallog. independent P nuclei are observed, with the magnitude of the 197Au quadrupole coupling being sufficiently large to produce a collapse of $1 \mathrm{~J}(\mathrm{Au}-\mathrm{P})$ splitting from quartets to doublets. The results highlight the important role played by the counteranion in the detection of the structural and spectroscopic properties of these sterically crowded d10 complexes.

\section{Catalysis}

\subsection{Carbon-Supported Gold Catalyst for Oxidation of Alcohols, Aldehydes, Polyhydroxy Compounds and Carbohydrates}

A supported gold catalyst on a doped with 0.01 - 1 wt.\% metal oxides porous carbon support used for oxidation of alcs., aldehydes, poly hydroxy compounds and carbohydrates is prepared by treating a porous support with an aq. soln. $\mathrm{HAuCl} 4$ in $0.1-12 \mathrm{~mol} / \mathrm{L}$ acids followed by drying at 60 - 200.degree. and reducing the catalyst during 10 - 300 min with $\mathrm{H} 2$ at .gtoreq.250.degree.. Patent No WO 2008095629, A1, 14th August 2008 Inventors A Haji Begli, C Baatz, N Decker, U Pruesse, K Vorlop, from Suedzucker Aktiengesellschaft Mannheim/ Ochsenfurt, Germany. Thus, gold nanoparticles having particle size 1 - $10 \mathrm{~nm}$ on a porous carbon support (gold content 0.1 - 5 wt.\%) were used for liq.-phase oxidation glucose to gluconic acid at 40.degree. exhibiting 100\% selectivity for aldehyde (C1) position glucose.

\subsection{Kinetic Evaluation of Highly Active Supported Gold Catalysts Prepared from Monolayer- Protected Clusters: An Experimental Michaelis- Menten Approach for Determining the Oxygen Binding Constant during $\mathrm{CO}$ Oxidation Catalysis}

Thiol monolayer-protected Au clusters (MPCs) were prepared using dendrimer templates, deposited onto a high-surface-area titania, and then the thiol stabilizers were removed under $\mathrm{H}_{2} / \mathrm{N}_{2}$. The resulting Au catalysts were characterized with transmission electron microscopy, XPS, and IR spectroscopy of adsorbed CO. C Long, J Gilbertson, G Vijayaraghavan, K Stevenson, C Pursell, B Chandler from the Departments of Chemistry, Trinity University, San Antonio, TX, USA, Journal of the American Chemical Society, 2008, 130(31), 10103. The Au catalysts prepared via this route displayed minimal particle agglomeration during the deposition and activation steps. Structural data obtained from the phys. characterization of the Au catalysts were comparable to features exhibited from a traditionally prepared std. Au catalyst obtained from the World Gold Council (WGC). 
A differential kinetic study of CO oxidation catalysis by the MPC-prepared Au and the std. WGC catalyst showed that these two catalyst systems have essentially the same reaction order and Arrhenius apparent activation energies $(28 \mathrm{~kJ} / \mathrm{mol})$. However, the MPC-prepared Au catalyst shows $50 \%$ greater activity for CO oxidation using a MichaelisMenten approach, the oxygen binding constants for the two catalyst systems were detected and found to be essentially the same within experimental error. To our knowledge, this kinetic evaluation is the first experimental detection of oxygen binding by supported Au nanoparticle catalysts under working conditions. The values for the oxygen binding equil. const. obtained from the MichaelisMenten treatment (ca. 29-39) are consistent with ultrahigh-vacuum measurements on model catalyst systems and support d. functional theory calculations for oxygen binding at corner or edge atoms on Au nanoparticles and clusters.

\subsection{Preparation of manganese oxide-cerium oxide-supported nano-gold catalyst and the application thereof}

This present invention provides the preparation of a manganese oxide-cerium oxide-supported nano-gold catalyst and a process for subjecting carbon monoxide and oxygen to interaction resulting in the formation of carbon dioxide in a hydrogen-rich environment by a manganese oxide-cerium oxide-supported nano-gold catalyst to remove carbon monoxide in hydrogen stream. Y-W Chen, M-H Lin, H-C Hsu, J-H Lin from the Tatung Company, Taiwan, Patent No US 20080193354, A1, 14th August 2008. The size of the nano-gold particle is less than $5 \mathrm{~nm}$ and supported on mixed oxides $\mathrm{MnO}_{2} / \mathrm{CeO}_{2}$ in various molar ratios. Preferential oxidation of $\mathrm{CO}$ in the presence of $\mathrm{CO}, \mathrm{O}_{2}$ and $\mathrm{H}_{2}$ by the manganese oxidecerium oxide-supported nano-gold catalyst is carried out in a fixed-bed reactor in the process of the present invention. The $\mathrm{CO} / \mathrm{O}_{2}$ molar ratio is in the range of 0.5 to 3. The manganese oxide-cerium oxide-supported nanogold catalyst of the present invention is applied to reduce CO concentration in hydrogen steam to less than 100 ppm to prevent CO from contaminating the electrodes of a fuel cell.

\subsection{Mercury oxidation in flue gas using gold and palladium catalysts on fabric filters}

The feasibility of oxidizing elemental $\mathrm{Hg}$ in coal combustion flue gas using a catalyst-impregnated fabric filter was examined $\mathrm{TiO}_{2}, \mathrm{Au} / \mathrm{TiO}_{2}$, and $\mathrm{Pd} / \mathrm{Al}_{2} \mathrm{O}_{3}$ were assessed based on promising results of previous research. Several fabric coating methods were examined to detect the best way to load the filter. J Hrdlicka, W Seames, M Mann, D Muggli, C Horabik from the Department of Chemical Engineering, University of North Dakota, Grand Forks, USA, Environmental Science \& Technology, 2008, 42(17), 6677.
A spray coat method had the highest initial loading and the lowest losses after simulated pulse-jet cleaning. Oxidation performance of catalyst-coated filters was tested with simulated flue gas in a bench-scale reactor under conditions similar to those in a baghouse. $\mathrm{Au} / \mathrm{TiO}_{2}$ and $\mathrm{Pd} / \mathrm{Al}_{2} \mathrm{O}_{3}$ were effective, yielding $\mathrm{Hg}$ oxidation of 40 $60 \%$ and $50-80 \%$, respectively. A baghouse-equipped 19 $\mathrm{kW}$ research combustor was used to fire a range of coals and further test $\mathrm{Pd} / \mathrm{Al}_{2} \mathrm{O}_{3}$ performance. Results warrant further development of this technique for $\mathrm{Hg}$ air pollution control.

\subsection{Alternative synthetic methods through new developments in catalysis by gold}

This review covers alternative gold catalyzed synthetic methods published until Nov. 2007 in both heterogeneous and homogeneous catalysis. A Arcadi from the Department of Chemistry, Chemical Engineering and Materials, University of L'Aquila, Coppito, Italy, Chemical Reviews (Washington, DC, United States), 2008, 108(8), 3266.

\subsection{Gold supported on mixed oxides for the oxidation of carbon monoxide}

$\mathrm{Au}$ catalysts (.apprx.1\%) supported by $\mathrm{CeO}_{2}, \mathrm{CeO}_{2}$ modified by included La (10\%) or Fe (5\%), and $\mathrm{CeZrO}_{4}$, were prepared, characterized (N2 physisorption, x-ray diffraction), and tested for CO oxidation (200-300.degree. K); stability tests for .Itoreq.75 h (Salford) and $24 \mathrm{~h}$ (Delft) were also performed. All 3 modified catalysts exhibited 10-20\% conversion at .apprx.200-243.degree. K, but La and $\mathrm{Zr}$ modifiers led to slightly decreased activity at 243293.degree. K. Au/La-CeO 2 and $\mathrm{Au} / \mathrm{Fe}-\mathrm{CeO}_{2}$ in particular, however, show exceptional stability in life-tests at ambient temperature $\mathrm{F}$ Moreau, G Bond, B van der Linden, B Silberova, M Makkee, Michiel, Institute for Materials Research, University of Salford, Salford, Greater Manchester, UK, Applied Catalysis, A: General, 2008, 347(2), 208. In particular, $\mathrm{Au} / \mathrm{La}-\mathrm{CeO}_{2}$ and $\mathrm{Au} / \mathrm{Fe}-\mathrm{CeO}_{2}$ displayed exceptional stability in life-tests at ambient temperature. Diffuse reflectance IR Fourier transform spectroscopy results for fresh and used catalysts suggested this may be due to better retention of surface hydroxide groups and lesser formation of $\mathrm{CO}_{32}-\mathrm{HCO}_{3}$ - near Au particles during reaction. Mechanisms to account for this behavior are considered; $\mathrm{OH}$ - groups acting catalytically may be responsible for stable activity.

\subsection{A comparative study of the selective oxidation of $\mathrm{NH}_{3}$ to $\mathrm{N}_{2}$ over gold,silver and copper catalysts and the effect of addition of $\mathrm{Li}_{2} \mathrm{O}$ and $\mathrm{CeOx}$}

The selective oxidation of $\mathrm{NH}_{3}$ into $\mathrm{N}$ over $\mathrm{Cu}, \mathrm{Ag}$, and $\mathrm{Au}$ catalysts at room temperature to 400.degree. using different $\mathrm{NH}_{3}: \mathrm{O}_{2}$ ratios is described. M Lippits, A Gluhoi, 
B Nieuwenhuys, Leids Instituut voor chemisch onderzoek, Universiteit Leiden, Leiden, Netherlands, Catalysis Today $2008, \mathbf{1 3 7}\left(\mathbf{2 - 4 )}, 446\right.$. The effect of adding $\mathrm{CeOx}$ and $\mathrm{Li}_{2} \mathrm{O}$ on activity and selectivity is also discussed. Results showed $\mathrm{Cu}$ and $\mathrm{Ag}$ are very active and selective toward $\mathrm{N}_{2}$; however, multi-component catalysts, $\mathrm{M} / \mathrm{Li}_{2} \mathrm{O} / \mathrm{CeOx} / \mathrm{Al}_{2} \mathrm{O}_{3}$ (where $\mathrm{M}=\mathrm{Au}, \mathrm{Ag}, \mathrm{Cu}$ ) performed best. On all 3 metalcontg. catalysts, activity and selectivity was affected by particle size and interaction between metal particles and support.

\subsection{Low energy photosynthesis of gold-titania catalysts}

By identifying the Electron Partitioning Effect (EPE) as responsible for the large gold deposits usually formed in the conventional photodeposition method, a low energy UV light-based method for the preparation of comparatively high-activity gold-titania catalysts was developed. R Kydd, K Chiang, J Scott, R Amal, ARC Centre for Functional Nanomaterials, School of Chemical Sciences and Engineering, University of New South Wales, Sydney, Australia, Photochemical \& Photobiological Sciences 2007, $\mathbf{6}(\mathbf{8}), 829$. These materials were tested in the carbon monoxide (CO) oxidation reaction and returned markedly higher levels of activity at room temperature, when compared to catalysts prepared by the traditional photodeposition method. This is the first instance of using a light-mediated process for prepg. catalysts active for the CO oxidation reaction.

\subsection{Study on the preparation and catalytic activity of gold nanoparticles supported on $\mathrm{Fe}_{2} \mathrm{O}_{3}$}

$\mathrm{Au} / \mathrm{Fe}_{2} \mathrm{O}_{3}$ catalyst was prepared by the coprecipitation method. C Nguyen,T Trang; N Thi Minh Nguyet; L Quang Huan; N Xuan, D Nguyen, T Do, Q Tran, Q Nguyen, Inst. of Materials Science, Vietnamese Academy of Science and Technol., Vietnam, Tap Chi Hoa Hoc, 2007, 45(6), 671. The formation process of $\mathrm{Au} / \mathrm{Fe}_{2} \mathrm{O}_{3}$ was investigated by DTA, TGA, DrTCA, TEM, HRTEM and XRD. The results show that the gold particle size of the sample calcined at 300 . degree. $C$ is in the range $1.2-7.8 \mathrm{~nm}$. The catalytic oxidation of $\mathrm{CO}$ and $\mathrm{H}_{2}$ was detected.

\subsection{Gold nanoparticles: from preparation to catalytic evaluation}

The role of protecting agent in liq.-phase oxidation, with focus on polyvinylalc. (PVA), and a new class of protecting agents formed by polyhydroxylated compounds, in particular D-glucose, were presented. M Rossi, C Della Pina, E Falletta, R Matarrese, from the Department CIMA, University of Milano, Milan, Italy, Metal Nanoclusters in Catalysis and Materials Science: The Issue of Size Control (2008), 253-262. Editor(s): Corain, Benedetto; Schmid, Guenter; Toshima, Naoki. Publisher: Elsevier B.V., Amsterdam, Netherlands. The larger scale preparation of the $1 \% \mathrm{Au} / \mathrm{C}$ catalyst was done on request of the World Gold Council (WCC). It was found that the most active catalyst is caused by $2.8 \mathrm{~nm}$ particles attained in the $2 \mathrm{~g}$ scale preparations of Au on XC72R. In addition, there was an important effect of sulfur impurities that are present in XC72R and almost absent in X40S carbon. A quite high activity, comparable with enzymic systems, can be obtained with different carbons in the case of glucose oxidation. In this latter case, gold particle size and sulfur poisoning thus play concomitant effects influencing the reaction rate.

\subsection{Gold colloidal nanoparticles sized to be} suitable precursors for heterogeneous catalysts A review. Suitable gold sol preparations designed for catalytic purposes were reported. The observations revealed that various support have different effects on transferring of the metal particles. F Porta, L Prati from the Department CIMA, Centre of Excellence CIMAINA, INSTM Unit, University of Milano, Milan, Italy, Metal Nanoclusters in Catalysis and Materials Science: The Issue of Size Control, 2008, 355-360. Editor(s): Corain, Benedetto; Schmid, Guenter; Toshima, Naoki. Publisher: Elsevier B.V., Amsterdam, Netherlands. It was also emphasized that carbon coupling with polyvinyl alc. (PVA) represents the best choice, assuring a relatively large range of PVA/Au ratio and a good stability of gold particles. Furthermore, the significance of size control has been shown for the selective oxidation of glycerol; it was exhibited that by increasing particle size a high selectivity to glycerate has been reached at the expense of the consecutive oxidation of glycerate to tartronate.

\subsection{Reaction-relevant gold structures in the low temperature water-gas shift reaction on $\mathrm{Au}-\mathrm{CeO}_{2}$}

In situ XANES and EXAFS showed Au structural changes of low-content (<1\%Au) Au-ceria catalysts in $\mathrm{H}_{2} \mathrm{O}$-gas shift (WCS) reaction tests at 100 and 200.degree., and after heating the used catalysts in $\mathrm{O}$ at 150.degree. Au in the unused (400.degree.-calcined) material was atomically dispersed in Ce oxide. W Deng, A Frenkel, R Si, M FlytzaniStephanopoulos, Department of Chemical and Biological Engineering, Tufts University, Medford, MA, USA, Journal of Physical Chemistry C, 2008, 112(33), 12834. Under WGS reaction conditions, reduction of the oxidized $\mathrm{Au}$ species was observed, accompanied by gradual Au aggregation.

The Au-Au coordination no. is zero for the unused material, but increases with the reaction temperature to 6.5 .+-. 2.4 (after use at 100.degree.) and to 8.7 .+-. 1.5 (after 200.degree.) in a He gas mixt. contg. 5\% CO and $3 \% \mathrm{H}_{2} \mathrm{O}$. Another important parameter is the reaction gas composition which dets. the extent of Au-O reduction The lower the reduction potential of the reaction gas 
mixt., the more oxidized the Au is in the used catalyst, and the higher its activity. The maximum activity of Au$\mathrm{CeO}_{2}$ was that of the fully dispersed Au-O-Ce unused material. Loss of surface $\mathrm{O}$ took place during reaction, as measured by H2-TPR of the used samples and it was commensurate with the activity loss. Attempts to reoxidize and re-disperse the Au by heating in $\mathrm{O}$ at 150.degree. were not effective. However recovery of the surface $O$ amt. and re-dispersion of Au in ceria was possible after a 400.degree.- O treatment of both the 100.degree.- and 200.degree.- used catalyst samples, with concomitant recovery of the initial catalyst activity. These tests were conducted by consecutive $\mathrm{H}_{2}$-TPR/steady-state catalyst activity measurements in the same microreactor.

\section{Chemistry}

\subsection{Ligand exchange of Au25SG18 leading to functionalized gold clusters: spectroscopy, kinetics, and luminescence}

Ligand exchange offers an effective way to modify the properties of the recently prepared quantum clusters of $\mathrm{Au}$. To tune optical and photoluminescence properties of one of the most stable quantum clusters of Au, Au25SG 18 (SC-glutathione thiolate), the authors functionalized it by the exchange of -SC with functionalized -SG and with an altogether different ligand, namely, 3-mercapto-2-butanol (MB). E Shibu, M Muhammed, T Habeeb; T Pradeep, DST Unit on Nanoscience (DST UNS), Department of Chemistry and Sophisticated Analytical Instrument Facility, Indian Institute of Technology, Madras, India, Journal of Physical Chemistry C, 2008, 112(32), 12168.

The products were characterized by various techniques such as optical absorption (UV-visible), FTIR, NMR, x-ray photoelectron (XPS), and luminescence spectroscopies, mass spectrometry, and TG. Analyses of the TG data helped to establish the mol. composition of the products. Ligand exchange reaction was monitored by NMR spectroscopy, and the exchange reaction follows a 1st order kinetics. The XPS study showed that after the exchange reaction there was no change in the chemical nature of the metal core and binding energy values of $\mathrm{Au}$ $4 f 7 / 2$ and $4 f 5 / 2$, which are similar in both the parent and the exchanged products. Photoluminescence studies of these clusters, done in the aerated conditions, showed that the excitation spectrum of the MB-exchanged product is entirely different from the acetyl- and formylglutathione exchanged products. The inherent fluorescence and solid-state emission of these clusters were observed This intense emission allows optical imaging of the material in the solid state. The emission is strongly temperature dependent. The synthesis of a diverse variety of clusters and their chemical stability and intense luminescence offer numerous applications in areas such as energy transfer, sensors, biolabeling, and drug delivery.

\subsection{Is gold always chemically passive?}

When epoxy-amine liquid mixtures are applied onto metallic substrates (such as Al, Ti, Sn, Zn, Fe, Cr, Cu, Ag, Ni, and $\mathrm{Au}$ ), concomitant amine chemisorption and metallic surface dissolution occur, leading to organo-metallic complex formation. M Aufray, A Roche, Laboratoire des Materiaux Macromoleculaires (IMP/LMM), INSA de Lyon, Villeurbanne, France, Applied Surface Science, 2008, 254(7), 1936. The interphase formation was studied, using two different amines as hardener (IsoPhoroneDiAmine and DiEthyleneTriAmine). If the complex concentration within the liquid amine or epoxy-amine prepolymer was higher than its soly. limit, the complexes will crystallize. Sharp needle-like crystals were only observed with metalIPDA organo-metallic complexes. A lot of metals are widely used as reactive substrates with gold as a ref., which is considered chemical inert. It is misleading, since it will be shown in this article that gold reacts with amine, just as the other metals.

\subsection{A facile and template-free method to prepare mesoporous gold sponge and its pore size control}

A facile, one-step, template-free method is reported to tune .beta.-D-glucose-stabilized Au nanoparticles into 3-D network nanowires and thus mesoporous gold sponge on a large scale by precise control of the soln. $\mathrm{pH}$ in an aq. medium at room temperature. G Qin, J Liu, T Balaji, X Xu, H Matsunaga, Y Hakuta, L Zuo, P Raveendran, Key Laboratory for Anisotropy and Texture of Materials (Ministry of Education), Northeastern University, Shenyang, Peop. Republic of China, Journal of Physical Chemistry C, 2008, 112(28), 10352. The Au nanoparticles appear to undergo sequentially linear aggregation and welding initially, and then randomly cross link into self-supporting, three-dimensional networks with time. The mesoporous gold sponge thus formed, on a millimeter to centimeter scale, exhibits a high sp. surface area of $11.9 \mathrm{~m} 2 / \mathrm{g}$ and small pore sizes of 5-30 nm, as well as high thermal stability .Itoreq.120.degree., above which it undergoes sintering and reorganization into macroporous gold sponge with pore sizes of 1-4 .mu.m depending on annealing temperature but still with mesoporous walls/ filaments.

\subsection{Dynamic restructuring of gold surfaces during chemical reactions}

A review. Professor Jacques-Emile Dubois pioneered the field of mol. visualization using mol. models constructed from experimental data. C Friend, Department of Chemistry and Chemical Biology, Harvard University, Cambridge, MA, USA, Actualite Chimique (2008), 320- 
321, 75. It is now possible to image and visualize atoms and mols. on solid surfaces using modern tools of surface chemical and physics. Contrary to expectations, seemingly immutable solids are teeming with activity on their surfaces. Atoms and mols. move around on the surface, even at room temperature. This article shows how species that bind to the surface from surrounding gases facilitate movement of surface atoms using gold surfaces to illustrate this phenomenon. The "dance" of atoms on surfaces is an essential property for important processes, such as catalytic chemical synthesis and use in microelectronic devices.

\section{Electrochemistry}

\subsection{Electroactive self-assembled monolayers on gold via bipodal dithiazepane anchoring groups}

Novel dithiazepane-functionalized ferrocenylphenylethynyl oligomers 1 and 2 were synthesized. P Bertin, D Georganopoulou, T Liang, A Eckermann, M Wunder, M Ahrens, G Blackburn, T Meade, Ohmx Corporation, Evanston, IL, USA, Langmuir, 2008, 24(16), 9096. Self-assembled monolayers (SAMs) of these ferrocene derivs. were studied by XPS, ellipsometry, and cyclic voltammetry. It was shown by XPS that monolayers of the dithiazepane-anchored mols. on Au electrodes contain Au-thiolate species. Cyclic voltammetry of the SAMs were characteristic of stable electroactive monolayers even for single-component SAMs of 1 and 2, with the more ideal responses recorded for the 2component SAMs dild. with undecanethiol. The small variation in peak splittings at progressively higher scan rates in these SAMs makes dithiazepane-bridged redox species promising candidates for further studies on mol. wires with bipodal anchoring.

\subsection{Electrochemical, spectroscopic and microscopic characterisation of novel poly(3,4-ethylenedioxythiophene)/gold nanoparticles composite materials}

Composite materials consisting of poly(3,4ethylenedioxithiophene) including $\mathrm{Au}$ nanoparticles encapsulated by bulky anionic species were electrogeneratedand characterized throughspectroscopic and microscopic techniques. F Terzi, C Zanardi, V Martina, L Pigani, R Seeber, Dipartimento di Chimica, Universita di Modena e Reggio Emilia, Modena, Italy, Journal of Electroanalytical Chemistry, 2008, 619-620, 75. The properties of electrodes modified by similar materials were studied with respect to the oxidation of different benchmark electroactive species, such as neg. charged ascorbic and uric acids, and positive charged dopamine. The responses obtained evidence the dependence of sensitivity and selectivity of the electrochemical responses on the presence and $d$. of the nanoparticles and on the nature of the relevant encapsulating agent. In particular, the insertion of neg. charged NPs activates a sort of electrostatic shield toward neg. charged mols.; however, also pos. charged species result to be conditioned in accessing the redox active centers, located either on the nanoparticle's surface or on the conducting polymer's mols.

\subsection{Shape-controlled gold nanoarchitectures: synthesis, superhydrophobicity, and electrocatalytic properties}

A simple 1-step electrodeposition method was used to fabricate various Au nanostructures on glassy C electrodes in a low concentration of $\mathrm{HAuCl}_{4}$ soln. (5 mM). H Zhang, $\mathrm{J} \mathrm{Xu}, \mathrm{H}$ Chen from the Key Laboratory of Analytical Chemistry for Life Science, School of Chemistry and Chemical Engineering, Nanjing University, Nanjing, Peop. Rep. China, Journal of Physical Chemistry C, 2008, 112(36), 13886. The morphologies of final Au nanostructures can be easily controlled by varying the $\mathrm{pH}$ of the precursors or the deposition temperature $\mathrm{X}$-ray powder diffraction, scanning electronic microscopy, TEM, contact angle measurements, and electrochemical methods were used to characterize them. Hierarchical waxberry-like $\mathrm{Au}$ nanostructures with high active surface areas were obtained in $\mathrm{pH} 4$ bath, and they had a higher catalytic performance for the reduction of oxygen than the other nanogold. These Au structures also displayed an extraordinary superhydrophobicity and the contact angle increased with the increase of deposition temperature and time. Their electrocatalytic response to the oxidation of glucose was also studied. A sensitive enzyme-free sensor can be easily developed for the detection of glucose in $\mathrm{pH} 7.4$ phosphate buffer solution.

\section{Electronics and sensors}

\subsection{Study of gold thin films evaporated on polyethylene naphthalate films toward the fabrication of quantum cross devices}

The authors have studied Au thin films evaporated on polyethylene naphthalate (PEN) org. substrates as a function of Au thickness < .apprx.20 nm and discussed its feasibility toward metal/insulator hybrid materials used for quantum cross devices using at. force microscope. H Kaiju, A Ono, N Kawaguchi, K Kondo, A Ishibashi, Laboratory of Quantum Electronics, Research Institute for Electronic Science, Hokkaido University, Sapporo, Japan, Materials Research Society Symposium Proceedings (2008), Volume Date 2007, 1025E(Nanoscale Phenomena in Functional Materials by Scanning Probe Microscopy), No pp. given, Paper1025-B07. The Au grain size increases 
from 28.0.+-.4.6 nm to 48.5.+-.11.4 nm with increasing the Au thickness from 6.9 to $20.8 \mathrm{~nm}$ and it denotes that the Au grain size is larger than its Au-thickness size, respectively. The surface roughness of Au films of sub-15$\mathrm{nm}$ thickness, in the scanning scale of the Au-thickness size, is less than $0.9 \mathrm{~nm}$, corresponding to 4-5 at. layers. These experimental results indicate that Au thin films on PEN substrates are suitable for possible metal/insulator hybrid materials to be used in quantum cross devices.

\subsection{Nanopatterning of gold colloids for label-free biosensing}

The authors present an approach for the controlled positioning of single gold colloids onto dot and line nanoarrays which have the potential to serve as label-free biosensor platforms. B Stadler, H Solak, S Frerker, K Bonroy, F Frederix, J Voros, M Grandin, Laboratory of Biosensors and Bioelectronics, Department of Information Technology and Electrical Engineering, Institute for Biomedical Engineering, ETH Zurich, Zurich, Switz. Nanotechnology ,2007, 18(15), 155306/1. The seperation between the high-resolution nanolithog. step, extreme UV interference lithog., and the subsequent functionalization has many advantages, among them the independence of the linkage chemical. The activation of the pre-patterned substrates is performed by transforming them into a surface of biotinylated nanopatches in a protein-resistant background. Complexes of streptavidin and singlestranded DNAs can then be selectively immobilized onto the biotinylated patches, which are embedded in an inert background. This approach enables the creation of single gold colloid dot and line arrays by directed self-assembly using the specificity of DNA hybridization.

\subsection{Optical properties of self-ensemble monolayers of gold metallic nanostructures}

This work analyzes the optical properties of nanostructures metallic surfaces of gold, at different sizes, in glass silanized substrates. Silanized substrates were immersed in the colloid soln. for one and half hour at room temperature and dried at room temperature for four hours. R Delgado Macuil, V Lopez Gayou, M Rojas Lopez, R Molina Contreras, J Garcia Servin, J Sanchez Ramirez, Centro de Investigacion en Biotecnologia Aplicada del IPN, Tlaxcala, Mex., AIP Conference Proceedings 2008, 992 (RIAO/OPTILAS 2007), 1242. The optical properties were analyzed by three spectroscopy techniques and by TEM microscopy. UV/VIS was used to observe changes in absorption due to the aminopropiltrimethoxysilane added to the glass substrate and due to the colloid added to silanized substrate. IR vibrational spectroscopy in ATR mode was used to observe the new generation peaks due to substrate silanization and SEIRA effect due to the colloid added to silanized substrate. And finally Z-Scan technique was used to observe the nonlinear properties of these funtionalization metallic nanostructures in function of nanostructures size. TEM images confirm the sizes observed in the UV/VIS spectra. (c) 2008 American Institute of Physics.

\subsection{Optical properties of core-shell gold-silver and silver-gold nanoparticles for some laser wavelengths}

Computer calculation of optical properties of core-shell metal nanoparticles was made for some laser wavelengths. V Pustovalov, K Koenig, L Astafyeva, W Fritzsche, Belarusian Institute of System Analysis, Minsk, 220004, Belarus, Proceedings of SPIE (2008), 6879 (Photon Processing in Microelectronics and Photonics VII), 687915/1. Efficiency factors of absorption, scattering and extinction by spherical core-shell gold-silver and silver-gold nanoparticles of the radii in the range 5-100 $\mathrm{nm}$ and for laser wavelengths 400, 532 and $800 \mathrm{~nm}$ were calculated. Analysis of influence of optical parameters of metals, radii of core and thicknesses of shell on optical properties of nanoparticles was made.

\section{Medical and dental}

\subsection{Highly efficient drug delivery with gold nanoparticle vectors for in vivo photodynamic therapy of cancer}

A highly efficient drug vector for photodynamic therapy (PDT) drug delivery was developed by synthesizing PEGylated gold nanoparticle conjugates, which act as a water-sol. and biocompatible "cage" that allows delivery of a hydrophobic drug to its site of PDT action. Y Cheng, A Samia, J Meyers, I Panagopoulos, B Fei, C Burda, Clemens, Center for Chemical Dynamics and Nanomaterials Research, Department of Chemistry, Case Western Reserve University, Cleveland, OH, USA, Journal of the American Chemical Society (2008), 130(32), 10643. The dynamics of drug release in vitro in a two-phase soln. system and in vivo in cancer-bearing mice indicates that the process of drug delivery is highly efficient, and passive targeting prefers the tumor site. With the Au NP-Pc 4 conjugates, the drug delivery time required for PDT has been greatly reduced to less than $2 \mathrm{~h}$, compared to 2 days for the free drug.

\subsection{A quantitative study on the photothermal effect of immuno gold nanocages targeted to breast cancer cells}

Gold nanocages with an average edge length of 65 .+-. 7 $\mathrm{nm}$ and a strong absorption peak at $800 \mathrm{~nm}$ were conjugated with monoclonal antibodies (anti-HER2) to target breast cancer cells (SK-BR-3) through the epidermal growth factor receptor (in this case, HER2), which is overexpressed on the surfaces of the cells. L Au, D; Zheng, 
F Zhou, Z Li, X Li, Y Xia,Department of Chemistry, University of Washington, Seattle, WA, 98195, USA, ACS Nano (2008), 2(8), 1645. Both the no. of immuno Au nanocages immobilized per cell and the photothermal therapeutic effect were quantified using flow cytometry. The targeted cells were irradiated with a pulsed near-IR laser, and by varying the power $d$., the duration of laser exposure, and the time of response after irradn., we were able to optimize the treatment conditions to achieve effective destruction of the cancer cells. We found that cells targeted with the immuno Au nanocages responded immediately to laser irradn. and that the cellular damage was irreversible at power densities greater than $1.6 \mathrm{~W} /$ $\mathrm{cm}_{2}$. The percentage of dead cells increased with increasing exposure time up to 5 min and then became steady. By quantifying the photothermal effect of immuno Au nanocages, crit. information with regards to both the optimal dosage of nanocages and parameters of the laser irradn. has been garnered and will be applied to future in vivo studies.

\subsection{Gold nanoparticles in delivery applications}

A review. P Ghosh, G Han, M De, C Kim, V Rotello, Department of Chemistry, University of Massachusetts, Amherst, MA, USA, Advanced Drug Delivery Reviews (2008), 60(11), 1307. Gold nanoparticles (AuNPs) provide non-toxic carriers for drug and gene delivery applications. With these systems, the gold core imparts stability to the assembly, while the monolayer allows tuning of surface properties such as charge and hydrophobicity. An additional attractive feature of AuNPs is their interaction with thiols, providing an effective and selective means of controlled intracellular release.

\subsection{High frequency of contact allergy to gold in patients with endovascular coronary stents}

Background: Stent implantation is an effective method for treatment of atherosclerotic disease. Factors predisposing to in-stent restenosis are still largely unknown. Contact allergy to metal ions eluted from the stent has been suggested to be a risk factor. Objectives: $\mathrm{S}$ Ekqvist, C Svedman, H Moeller, M Kehler, C Pripp, J Bjoerk, B Gruvberger, E Holmstroem, C Gustavsson, M Bruze, Departments of Occupational and Environmental Dermatology, Malmoe University Hospital, Lund University, Malmoe, 205 02, Sweden, British Journal of Dermatology (2007), 157(4), 730. To explore whether there is a possible induction of contact allergy to metals used in stents among patients with a stainless steel stent contg. nickel (Ni stent) and patients with a gold-plated stent (Au stent).

Methods: Adults ( $n=484$ ) treated with coronary stent implantation participated in the study with patch testing. The study design was retrospective and cross-sectional with no assessment of contact allergy before stenting.
Age- and sex-matched patch-tested patients with dermatitis ( $n=447)$ served as controls. Results: Of Austented patients, 54 of 146 (37\%) were allergic to gold compared with 85 of $447(19 \%)$ controls $(P<0$. cntdot.001). Within the stented population there were no statistically significant differences in contact allergy to gold or nickel between Ni-stented and Au-stented patients. In multivariate models where other risk factors for contact allergy to gold were considered, the Au stent showed a trend towards statistical significance (odds ratio 1.cntdot.43, 95\% confidence interval 0.cntdot.95-2. cntdot.16; $P=0 . c n t d o t .09)$. Conclusions: As the frequency of contact allergy to gold is higher in stented patients independent of stent type it suggests a previous sensitization. However, several pieces of circumstantial evidence as well as statistical anal. indicate the possibility of sensitization in the coronary vessel by the Au stent. Ni stents and Au stents should not be ruled out as risk factors for induction of contact allergy to these metals.

\subsection{Application of gold nanoparticles for targeted therapy in cancer}

A review. Cancer is a major public health problem worldwide. Some of the key challenges in cancer are early detection, diagnosis, and targeted therapy. Most of the patients are usually diagnosed in the late stage of the disease causing immunity to therapeutic intervention. Another major challenge in the therapeutic intervention of cancer is the toxicity of the anti-cancer drugs. When administered, these drugs are not only toxic towards malignant cells, but also towards normal cells. Nanotechnology can play a pivotal role in changing the very foundation of cancer treatment and diagnosis. At the heart of the nanotechnol. are the nanoparticles and they can be synthesized in different shapes and sizes with tailor able optoelectronic and physico-chemical properties.

Due to very high surface area of these nanoparticles, a no. of biomols. can be loaded to create a nanobioconjugate with multifunctional capabilities. Such a bi-functional nanobioconjugate contg. a targeting agent and a drug would lead to targeted delivery of the drug to the malignant cells with better therapeutic efficacy and with reduced systemic toxicity. In a similar fashion targeted delivery of an imaging agent could lead to early detection of cancer. The design and development of nanoparticles and its nanobioconjugates in conjunction with the development of genomics and proteomics to identify biomarkers raise exciting opportunities for personalized treatment. In this particular review, the authors have discussed application of gold nanoparticles (AuNPs) in cancer and the current standing with respect to nanotechnol. C Patra, R Bhattacharya, D Mukhopadhyay, P Priyabrata, Department of Biochemistry and Molecular Biology, Mayo Clinic, Rochester, MN, 55905, USA, Journal of Biomedical Nanotechnology (2008), 4(2), 99. 


\section{Metallurgy, materials and coatings}

7.1 Matrix effects on the surface plasmon resonance of dry supported gold nanocrystals

The Authors present a method to characterize surfacechemical properties of gold nanocrystals. Spherical, $60 \mathrm{~nm}$ gold nanocrystals were immobilized on quartz substrates by a coupling agent and cleaned in a hydrogen plasma. C Kuemin, T Kraus, H Wolf, N Spencer, Department of Materials, Laboratory of Surface Science and Technology,Department of Materials, ETH Zurich, Zurich, CH-8093, Switz.Optics Letters (2008), 33(8), 806. The nanocrystals were then functionalized with alkanethiol self-assembled monolayers (SAM) of varying chain lengths by adsorption from the gas phase, and localized surface plasmon resonance (LSPR) spectroscopy was performed on the samples. Depending on the alkanethiol chain length, the adsorption of the SAM red shifted the LSPR to different extents, in accordance with Mie theory. SAM thickness differences below $1 \mathrm{~nm}$ could be easily resolved. The results demonstrate that LSPR spectroscopy can be applied to characterize thin org. layers on dry supported gold particles with high sensitivity.

\subsection{Transformed gold island film improves light-to-heat transduction of nanoparticles on silica capillaries}

Gold (Au) nanoparticles (NP) assembled on inner walls of silica $\left(\mathrm{SiO}_{2}\right)$ capillaries dissipated .gtoreq.10-fold more heat (.gtoreq.96.92 .+-. 8.94 vs .Itoreq.9.92 .+-. 2.06\%) from incident photons via localized surface plasmon resonance (LSPR) with a 10-fold faster response time (.toreq.8.39 .+-. 1.05 vs .gtoreq.86.30 .+-. 8.61 s) than colloidal Au NPs suspended in $\mathrm{H}_{2} \mathrm{O}$. Au NP assemblies were created by thermal transformation of gold island film (TGIF) that was electrolessly plated on the inner capillary wall to form an optical plasmon capillary (OPC). W Ahn, D Roper, Department of Materials Science and Engineering, University of Utah, Salt Lake City, UT, USA, Journal of Physical Chemistry C 2008, 112(32), 12214. Enhanced laser-to-heat transduction of the solid-state Au NP OPC resulted from the ability to tune the LSPR peak to the laser line, elimination of laser- or heat-induced NP aggregation, and enhanced thermal transport of the Au NP assembly on the OPC. This yielded .gtoreq.6.4-fold higher temperature increase per unit incident laser power (157.07 .+-. 21.68 vs 24.51 .+-. 4.45 .degree.C watt-1) relative to suspended Au NPs. Thermal behavior of the OPC was predictable using design equations obtained from an overall energy balance and consistent with nanoscale opto-thermal expressions.

\subsection{Growth of two-dimensional arrays of uncapped gold nanoparticles on silicon substrates}

A method of preparing large area patterned 2D arrays of uncapped Au nanoparticles was developed. The pattern was formed using self-assembly of uncapped Au nanoparticles. A Das, S Das, A Raychaudhuri, DST Unit for Nanoscience, S.N. Bose National Centre for Basic Sciences, Kolkata, 700 098, India, Bulletin of Materials Science, 2008, 31(3), 277. The Au nanoparticles were synthesized via toluene/water 2 phase systems using a reducing agent and colloidal soln. of Au nanoparticles was produced. These nanoparticles were prepared without using any kind of capping agent. Analysis by TEM showed discrete Au nanoparticles of $4 \mathrm{~nm}$ average diameter AFM analysis also showed similar result. The TEM studies showed that these nanoparticles formed self-assembled coherent patterns with dimensions exceeding 500 nm. Spin coating on Si substrate by suitably adjusting the speed can self-assemble these nanoparticles to lengths exceeding 1 .mu.m.

\subsection{Synthesis of gold nanosheets at a liquid/liquid interface using an amphiphilic polyoxometallate/ surfactant hybrid photocatalyst}

Control of the morphology of gold nanoparticles has received considerable attention because the phys. and chemical properties of gold depend significantly on its size and shape. T Kida from the Department of Energy and Material Sciences, Faculty of Engineering Sciences, Kyushu University, Kasuga-shi, Fukuoka, 816-8580, Japan, Langmuir, 2008, 24(15), 7648. A novel route for obtaining 2-D gold nanostructures has been developed in which chloroaurate ions $\left(\mathrm{AuCl}_{4}\right)$ are reduced at the 2-D interface between water and chloroform using an amphiphilic polyoxometallate (SiW120404-)/ surfactant (dimethyldioctadecylammonium; DODA) hybrid photocatalyst under UV irradn. at room temperature in air. This simple method can readily produce large single-cryst. gold nanosheets (lateral size, ca. 20 .mu.m; thickness, ca. $150 \mathrm{~nm}$ ).

\subsection{Alloying effects of cosputtered gold-platinum thin films on the oxygen reduction reaction in acidic electrolyte}

A better understanding of the effects of alloying Pt with Au on the oxygen reduction reaction (ORR) was achieved. Recent studies of C-supported Au-Pt nanoparticle catalysts showed enhanced response for the ORR with respect to pure Pt nanoparticles. B Brown; S Wolter, B Stoner, J Glass, Department of Electrical and Computer Engineering, Duke University, Durham, NC, 27708, USA, Journal of the Electrochemical Society, 2008, 155(8), B852. These results were reported to be due to homogeneous mixing of $\mathrm{Au}$ and Pt atoms that was accomplished in nanoscale particles, effectively removing the miscibility gap inherent in the equil. phase diagram. Cosputtering of Au and Pt is used to form $350 \mathrm{~nm}$ thick films to achieve homogeneous mixing without nanoparticle geometries, thereby isolating the effects of alloying from nanoparticle effects. ORR 
activity and stability at these electrodes are studied via cyclic voltammetry in $\mathrm{O}_{2}$-satd. $0.5 \mathrm{M} \mathrm{H}_{2} \mathrm{SO}_{4}$ under oxidizing potentials. Cosputtering yielded solid solution alloys throughout the entire composition range as shown by $\mathrm{x}$ ray diffraction results. Also, as-deposited near-surface compns. detected by XPS indicated that extensive Au surface segregation was avoided by room-temperature deposition. Metastable compns. within the miscibility gap, explicitly Pt21Au79, Pt43Au57, and Pt59Au41, are significantly more electrocatalytic for $\mathrm{O}_{2}$ reduction than the pure Pt film, although Pt43Au57 and Pt59Au41 rapidly lost catalytic activity with subsequent potential sweeps.

\subsection{Gold- $\mathrm{Ga}_{2} \mathrm{O}_{3}$ Co-shell nanowires}

A simple thermal annealing process was carried out to produce the innovative heterostructure of gold- $\mathrm{Ca}_{2} \mathrm{O}_{3}$ co-shell nanowires. C Hsieh, L Chou, Department of Materials Science and Engineering, National Tsing Hua, University, Hsinchu, 300, Taiwan, ECS Transactions, 2007, 11(8), 45. By changing the growth temperature and the size of the gold catalyst, it is easy to control the growth of pure $\mathrm{Ga}_{2} \mathrm{O}_{3}$ or gold- $\mathrm{Ga}_{2} \mathrm{O}_{3}$ co-shell nanowires. The twin boundary induced gold- $\mathrm{Ga}_{2} \mathrm{O}_{3} \mathrm{co}$-shell nanowires, including the gold-peapodded and gold- $\mathrm{Ca}_{2} \mathrm{O}_{3}$ core-shell nanowires, were first fabricated by the reaction of the gold, gallium metal and silica. The morphologies, crystal structures and microstructures were investigated by field-emission scanning electron microscope, $x$-ray spectrometer and field-emission transmission electron microscope, respectively. Furthermore, the novel 1-D gold- $\mathrm{Ga}_{2} \mathrm{O}_{3}$ co-shell nanowires shows the potential application for the next generation optoelectronic devices in semiconductor industry.

\section{Nanotechnology}

\subsection{Reaction dynamics for gold nanoparticles synthesis in solution plasma}

The authors describe the dynamics of the synthesis of gold nanoparticles by glow discharge in aqueous. solns. Initial $\left[\mathrm{AuCl}_{4}\right]^{-}$concentration and the voltage applied between the electrodes were varied. J Hieda, N Saito, O Takai, Osamu, Department of Materials, Physics and Energy Engineering, Graduate School of Engineering, Nagoya University, Nagoya, 464-8603, Japan, Materials Research Society Symposium Proceedings, 2007, 1056E (Nanophase and Nanocomposite Materials V), No pp. given, Paper 1056-HH03. Reduction rates were calculated from changes in concentration of $\left[\mathrm{AuCl}_{4}\right]^{-}$vs. discharge time. A pulsed power supply was used to generate discharges in the aqueous solutions. The morphology of the nanoparticles obtained was observed by transmission electron microscopy (TEM). $\left[\mathrm{AuCl}_{4}\right]^{-}$was reduced by $\mathrm{H}$ radicals or electrons generated by the discharge.
Dendrite-shaped nanoparticles .apprx.150 nm in size were formed after discharge for $1 \mathrm{~min}$. The $\mathrm{pH}$ of the solution decreased gradually with increasing discharge time. The decrease in $\mathrm{pH}$ led to dissolution of gold nanoparticles. The reduction and dissolution rates increased proportionately with the applied voltage. The size of gold nanoparticles decreased during discharge and was $20 \mathrm{~nm}$ after discharge for $45 \mathrm{~min}$. When the reduction rate lowered as a result of dissolution, anisotropic nanoparticles were formed and continued to grow in the solution.

\subsection{Optical cavity modes in gold shell colloids}

Core-shell colloids composed of a dielec. core surrounded by a metal shell show geometric cavity resonances with optical properties that are distinctly different than those of the collective plasmon modes of the metal shell. J Penninkhof L Sweatlock, A Moroz, H Atwater, A van Blaaderen, A Polman, Center for Nanophotonics, FOM Institute for Atomic and Molecular Physics, (AMOLF), Amsterdam, 1098 SJ, Neth., Journal of Applied Physics, 2008, 103(12), 123105/1. The authors use finitedifference time domain calculations on silica colloids with a core diameter of $456 \mathrm{~nm}$, surrounded by a $38 \mathrm{~nm}$ thick Au shell, to study the temporal evolution of the mode field intensity inside the cavity upon pulsed excitation. Calculations using Mie theory and the T-matrix method are used to anal. detect the dipolar cavity resonance spectrum, which is found superimposed on the broad collective dipolar plasmonic resonance modes. They characterize resonance wavelength and linewidth in terms of a geometric mode confined inside the cavity. Cavity linewidth can be controlled by metal shell thickness and quality factors Q.degree.150 are observed Due to the small cavity mode vol. $V=0.2($.lambda./n)3, a Purcell factor as high as $\mathrm{P}=54$ is calcd. Introducing shape anisotropy lifts the cavity mode degeneracy, yielding blue- and red-shifted longitudinal and transverse resonant modes, respectively. The relatively large vol. over which the field enhancement is observed in these spherical and anisotropic metal shell cavities, combined with cavity quality factors that are much higher than that of the collective plasmonic modes, makes them attractive for application in nanoscale light sources, sensors, or lasers. (c) 2008 American Institute of Physics.

\subsection{Synthesis of flexible, ultrathin gold nanowires in organic media}

Gold nanoparticles are very interesting because of their potential applications in microelectronics, optical devices, anal. detection schemes, and biomedicine. N Pazos-Perez, D Baranov, S Irsen, M Hilgendorff, L Liz-Marzan, M Giersig, Department of Nanoparticle Technology and Electron Microscopy Group, Center of Advanced European Studies and Research (CAESAR), Bonn, 53175, Germany, Langmuir 
(2008), 24(17), 9855. Though shape control has been achieved in several polar solvents, the capability to prep. organosols contg. elongated gold nanoparticles has been very limited. In this work we report a novel, simplified method to produce long, thin gold nanowires in an org. solvent (oleylamine), which can be readily redispersed into nonpolar org. solvents. These wires have a characteristic flexible, hairy morphol. arising from a small thickness ( $<2$ $\mathrm{nm}$ ) and an enormous length (up to several micrometers), with the possibility of adjusting the dimensions through modification of the growth conditions, in particular, the gold salt concentration Despite their extreme aspect ratio, the wires are stable in soln. for long periods of time but easily break when irradiated with high-energy electron beams during transmission electron microscopy.

\section{4 $\mathrm{pH}$ Tunable morphology of the gold nanoparticles produced by citrate reduction}

The reduction of $\mathrm{AuCl}^{4-}$ by citrate produces $\mathrm{Au}$ nanoparticles with a variety of shapes and sizes depending on the exact conditions used in the preparation. The $\mathrm{pH}$ of the reacting mixture has a dramatic effect on the size, polydispersity, and morphol. of the resulting $\mathrm{Au}$ nanoparticles. W Patungwasa, J. Hodak, Center for Nanoscience, Faculty of Science, Mahidol University, Phayathai, Bangkok, 10400, Thailand, Materials Chemistry and Physics 2008, 108(1), 45. This occurs by altering the citrate charge controlling its stabilizing effect during the growth of the particles. On one hand, wide size distributions of predominantly polyhedra are obtained at $\mathrm{pH}<5.0$. On the other hand, narrow size distributions of nearly spherical particles are obtained at $\mathrm{pH}>6$. A mixture of ellipsoidal and other shapes with aspect ratio larger than one is formed when the reaction is performed at a $\mathrm{pH}$ in the 5-6 range. The mean particle diams. as well as the width of the distributions decrease monotonically as the $\mathrm{pH}$ of the solution increases settling to the wellknown values for these colloids with a width of. apprxeq. $10 \%$ of the mean diameter The variation in the size distribution is controlled mainly by the average charge per citrate mol.

\subsection{Surface tension and sintering of free gold nanoparticles}

Processes such as coalescence, sintering, and evapn. depend on the surface tension. We have evaluated the surface tension of free gold nanoparticles from the sizedependent evapn. data. K Nanda, A Maisels, F Kruis, Materials Research Centre, Indian Institute of Science, Bangalore, India, Journal of Physical Chemistry C (2008), 112(35), 13488. A value of $8.78 \mathrm{~N} / \mathrm{m}$ has been obtained, which is in very good agreement with other theor. models, and the values deduced from the size-dependent lattice parameter. The value of surface tension on the sintering process of gold nanoparticles is discussed.

\section{Refining}

\subsection{An investigation on reusing process water in gold cyanidation}

Water is used extensively in gold processing. The reuse of water is encouraged both to conserve water and to minimize the discharge of contaminated wastewater into surface streams. However, the use of recycled process water can neg. affect gold and silver recovery. In the present lab. study, the authors compared $\mathrm{Au}, \mathrm{Ag}$, and $\mathrm{Hg}$ recoveries when leaching with fresh water, original process water, and process water that had been either neutralized or treated with activated carbon to remove the dissolved metals. A Bahrami, M Hosseini, K Razmi, Department of Mining Engineering, Urmia University, Urmia, Iran, Mine Water and the Environment (2007), 26(3), 191. Recovery of $\mathrm{Au}$ and $\mathrm{Ag}$ were 93 and $71.7 \%$ in leaching with fresh water, and 91 and $66.2 \%$ in leaching with process water that been treated with activated carbon for $1 \mathrm{~h}$. Cyanide consumption was substantially reduced when process water was used, indicating that using process water is feasible.

\subsection{Application of smelting process for gold extraction}

The smelting process was presented for extracting gold from preciptates, ashing of gold-loaded activated carbon and high grade concentrations produced by middle-small scale gold producer in Xinjiang Aletai area. W Wang, Y Lu, Y Wang, No.4 Geological Team of Xinjiang Geology Mineral Bureau, Peop. Rep. China, Huangjin (2006), 27(7), 36. Application of the smelting process in prodn. for years demonstrated recovering gold economically, gaining gold extraction of $99 \%$ and product gold purity of over 999 . permill.

\subsection{Investigations on acidic hot-pressing pre- oxidation of refractory gold concentrates}

The gold recovery can be increased from 35.6\% to $94.3 \%$ by pretreating refractory gold concentrations by acidic hot-press oxidation process. H-G Huang, Huai-guo from the Research Institute of Mining and Metallurgy, Zijin Mining Group Co Ltd,Shanghang, Fujian, Peop. Rep. China, Kuangye Gongcheng (Changsha, China) 2007, 27(4), 42. The effect of temperature, retention time, pulp d., oxygen partial pressure and particle size on recovery was studied.

\subsection{Experimental research on extracting technologies of gold from a refractory ore containing sulfur and arsenic}

Under the conditions of using conventional cyanide leaching and thiourea leaching technologies, the leaching rates of gold from flotation conc. of the refractory ore contg. sulfur and arsenic are both low. The leaching rates 
of gold are $31.52 \%$ and $33.71 \%$, resp. Q Xia, Y Yin, J Nie, X Fang, $T$ Qiu, from the Jiangxi University of Science and Technology, Ganzhou, Jiangxi, Peop. Rep. China, Kuangchan Zonghe Liyong (2007), (5), 3. To this end, the exptl. research on biooxidn. pretreatment was performed. The conditions used in the thiourea leaching of gold were explored, and the research on leaching gold with thiourea promoted by magnetic field was carried out. The results showed that by using the combined technol. of biooxidn. pretreatment and thiourea leaching of gold intensified by magnetic field, the leaching rate of gold reached $90.06 \%$.

\subsection{Recovery of high purity gold and silver using mesoporous adsorbents}

Mesoporous silica contg. amino surface groups displayed good capacity and excellent selectivity for adsorption of precious metals (i.e., Au and Ag). K Lam, C Fong, Y Yeung, G McKay from Department of Chemical Engineering, The Hong Kong University of Science and Technology, Kowloon, Hong Kong, Studies in Surface Science and Catalysis 2007, 170B (From Zeolites to Porous MOF Materials), 1969-1974. The adsorbed metals were recovered as high purity metal salt solution by simple acid wash and the regenerated adsorbents were reused without lost of performance.

\subsection{Evaluating an alternative to the gravity recoverable gold (grg) test}

Knelson concentrators are commonly used to recover fine liberated gold in gold processing plants. G Subasinghe, Department of Mineral Science and Extractive Metallurgy, Murdoch University, Perth, WA, 6000, Australia, Publications of the Australasian Institute of Mining and Metallurgy (2007), 1/2007 (Ninth Mill Operators' Conference, 2007), 235. The decision to employ a gravity gold circuit consisting of centrifugal devices such as Knelsons is primarily taken on the results of GRG tests, among other considerations. While the GRG test is considered a material characterization test, which is useful in the design stages, its use in evaluating the performance of an existing unit is questionable. The problem is exacerbated by the fact that it is time consuming and costly. This paper examines the validity of the GRG test for performance evaluation and discusses alternative test work that can be performed to evaluate the performance characteristics of an operating machine. Principles that are useful in selecting optimal operating parameters, such as optimal fluidising water flow rate for a given ore/machine combination, are also discussed and illustrated with lab. and plant data. 